\title{
Patologías tratadas con oxigenoterapia hiperbárica en el Hospital Central de la Defensa
}

\author{
Torres León JM. ${ }^{1}$, Domínguez Alegría AR. ${ }^{2}$, Navarro Téllez M. ${ }^{3}$, Brinquis Crespo MA. ${ }^{4}$, \\ Espigares Correa A. ${ }^{2}$, Pérez Mochales JF. ${ }^{5}$
}

Sanid. mil. 2015; 71 (2): 77-83; ISSN: 1887-8571

\begin{abstract}
RESUMEN
Introducción: La oxigenoterapia hiperbárica $(\mathrm{OHB})$ es una modalidad terapéutica que se fundamenta en la obtención de presiones parciales de oxígeno elevadas en sangre, al respirar oxígeno puro, en el interior de una cámara hiperbárica a una presión superior a la atmosférica. Sus acciones incluyen efectos hemodinámicos, acciones sobre la inmunidad y el transporte de O2. Este amplio espectro de efectos facilita que sus recomendaciones puedan incluir una gran variedad de indicaciones, algunas de ellas controvertidas. Objetivos: Conocer las patologías de los pacientes tratados con OHB en la Comunidad Autónoma de Madrid (CAM) y revisar la evidencia científica al respecto. Método: En la actualidad la cámara hiperbárica del Servicio de MSB (Medicina Subacuática) del HCD (Hospital Central de la Defensa) es la de referencia en la CAM. Se revisaron las historias clínicas de los pacientes atendidos entre Febrero 2013 y Junio 2014. Se recogieron las siguientes variables: 1) Enfermos aceptados y desestimados para el tratamiento. 2) Edad y sexo de los pacientes tratados. 3) Patología por la que se indicaba el tratamiento. 4) Complicaciones observadas en relación con la OHB. Resultados: Se aceptaron para tratamiento 113 enfermos procedentes de 15 Hospitales de la CAM. Se desestimaron para tratamiento con OHB a 22 pacientes por patologías o tratamientos activos que no hacían aconsejable el tratamiento con OHB en ese momento. El 59,82\% eran hombres y el 40,18 \% mujeres. Edad media 64,72 años. Las indicaciones principales de tratamiento fueron las lesiones radio inducidas 52,21 \% de los casos $(n=59)$ y las úlceras y heridas de evolución tórpida con mala respuesta a tratamiento convencional $23 \%$ de los casos $(n=26)$. El tratamiento tuvo que ser suspendido en 8 pacientes (7,14 \% de los tratados) por mala tolerancia o complicaciones leves. Discusión y conclusiones: Las indicaciones de $\mathrm{OHB}$ en nuestra muestra comprenden un amplio número de patologías. Las lesiones radio inducidas son la indicación más frecuente de los enfermos tratados con OHB en el HCD. En este grupo destaca la osteorradionecrosis de mandíbula (ORNM) tras radioterapia (RT) en tumores de cabeza y cuello. Todas las indicaciones de OHB de la muestra presentada cuentan con estudios que avalan su uso, aunque no existen para ninguna de ellas estudios randomizados controlados, doble ciego. Las complicaciones que observamos en el tratamiento con $\mathrm{OHB}$ en nuestra muestra son leves por lo que la podemos considerar un tratamiento seguro. Creemos que la $\mathrm{OHB}$ ofrece una gran oportunidad para investigar la evidencia científica firme que avale sus indicaciones en discusión.
\end{abstract}

PALABRAS CLAVE: Oxigenoterapia hiperbárica, Lesiones radio inducidas.

Pathologies treated with hyperbaric oxygen therapy in The Central Hospital of Defense

SUMMARY: Introduction: Hyperbaric oxygen therapy (HBOT) is a therapeutic modality that is based on obtaining high partial pressures of oxygen in blood, breathing pure oxygen inside a hyperbaric chamber at a pressure above atmospheric. Their actions include hemodynamic effects, actions on immunity and $\mathrm{O} 2$ transport. This broad spectrum of effects makes its recommendations may include a variety of indications, some of them controversial. Objectives: To know the pathologies of patients treated with HBO in the Autonomous Community of Madrid (CAM) and review the scientific evidence for this. Method: At present the hyperbaric chamber MSB Service (Underwater Medicine) HCD (Central Hospital of Defense) is the reference in the CAM. The medical records of patients treated between February and June-13-14 were reviewed. The following variables were collected: 1) Sick accepted and rejected for treatment. 2) Age and sex of patients treated. 3) Pathology for which treatment is indicated. 4) Complications observed in connection with HBO. Results: 113 patients accepted for treatment from 15 hospitals in the CAM. Were rejected for HBO therapy for pathologies 22 patients or active treatments did not advisable HBO therapy at that time. The 59.82\% were male and 40.18\% female. Mean age 64.72 years. The main indications for treatment were within lesions induced 52.21\% of cases $(\mathrm{n}=59)$ and $\mathrm{ulcers}$ and wounds torpid with poor response to conventional treatment $23 \%$ of cases $(n=26)$. The treatment had to be suspended in 8 patients $(7.14 \%$ treated) by poor tolerance or mild complications. Discussion and conclusions: The indications for HBO in our sample comprises a large number of pathologies. Radio induced injuries are the most common indication for patients treated with $\mathrm{HBO}$ in the HCD. In this group highlights the jaw osteoradionecrosis

Tcol. Médico. Servicio de Medicina Interna.

${ }^{2}$ Médico civil especialista. Servicio de Medicina Interna

${ }^{3}$ Cap. Médico en formación de especialidad (MAFE) en Medicina Interna.

${ }^{4}$ Cor. Médico. Servicio de Medicina Subacuática.

${ }^{5}$ Cor. Médico. Servicio de Medicina Interna.

Hospital Central de la Defensa Gómez Ulla. Madrid. España.

Dirección para correspondencia: Dr. Juan M. Torres. Servicio de Medicina Interna. Hospital Central de la Defensa "Gómez Ulla". Glorieta del Ejército 1. 28047 Madrid.

Recibido: 1 de octubre de 2014.

Aceptado: 12 de febrero de 2015.
(ORNM) after radiotherapy (RT) in head and neck tumors. All indications OHB sample have presented studies supporting its use, although there are none for randomized controlled trials, double-blind. The complications observed in the HBO treatment in our sample are mild so we can consider it a safe treatment. We believe that HBO offers a great opportunity to investigate the firm scientific evidence to support its indications discussion.

KEYWORDS: Hyperbaric oxygen therapy, Radio induced lesions. 


\section{INTRODUCCIÓN}

La OHB se define por tres elementos esenciales: a) la administración terapéutica de $\mathrm{O} 2, \mathrm{~b}$ ) a una presión mayor de la ambiental y c) en una cámara hiperbárica1. Es una modalidad terapéutica que se fundamenta en la obtención de presiones parciales de oxígeno elevadas en sangre, al respirar oxígeno puro, en el interior de una cámara presurizada ${ }^{2}$. La presión se mantiene por lo general entre 2,4 y 3 ATA, cada sesión dura entre 60 y 90 minutos, aunque puede prolongarse por mucho más tiempo en algunas situaciones como la enfermedad descompresiva grave. El número de sesiones varía de una a dos, en las indicaciones por procesos agudos, a 40 o más sesiones en las enfermedades crónicas ${ }^{3}$. Las cámaras pueden ser monoplaza o multiplaza, las primeras presurizadas con oxígeno puro y las segundas con aire comprimido. Las cámaras multiplaza tienen la ventaja de permitir la asistencia médica directa durante la sesión en el caso de enfermos críticos y su sistema de presurización hace que el riesgo de deflagración sea mucho menor si lo comparamos con el que presentan las cámaras monoplazas.

Los efectos fisiológicos de la OHB pueden explicarse a partir de las leyes que regulan el comportamiento de los gases y las consecuencias bioquímicas y fisiológicas de la hiperoxia.

La ley de Boyle establece que, a una temperatura constante, el volumen de un gas es inversamente proporcional a la presión. Esta ley explica el uso de la OHB en los accidentes por descompresión brusca y en las embolias gaseosas, con objeto de reducir el tamaño y disolver las burbujas de nitrógeno que son las responsables de estos procesos.

La ley de Dalton explica que la presión ejercida por un gas mixto es igual a la suma de las presiones parciales de los gases que forman la mezcla. La administración de oxígeno al $100 \%$, y a una presión superior a la atmosférica, hacen posible que la presión parcial del oxígeno inspirado sea muy superior a la del aire ambiente. En la intoxicación por $\mathrm{CO}$, un gas con una afinidad por la hemoglobina 200-250 veces mayor que la del oxígeno y que desplaza la curva de disociación de la hemoglobina a la izquierda, la OHB puede disminuir la vida media de la carboxihemoglobina de 4-5 horas a 15-30 minutos ${ }^{4}$. Además de favorecer la oxigenación a los tejidos por el mecanismo que se describe en el siguiente párrafo.

La ley de Henry establece que la solubilidad de un gas en un líquido es directamente proporcional a la presión que este ejerce en dicho líquido. La mayor parte del oxígeno es transportado unido a la hemoglobina y una pequeña proporción $(0,3 \mathrm{ml} / \mathrm{dl})$ lo hace disuelto en el plasma. El aumento de la presión parcial de oxígeno en sangre, producida al inhalarlo al $100 \%$ y a una presión de 3 ATAs, eleva el oxígeno disuelto en el plasma hasta unas 20 veces su valor normal. El oxígeno disuelto puede alcanzar áreas con bajo flujo sanguíneo, donde no son capaces de llegar los hematíes, y aliviar situaciones en las que el transporte de oxígeno por la hemoglobina se encuentra comprometido, como en casos graves de anemia o intoxicación por monóxido de carbono ${ }^{5}$.

La hiperoxia debida a la $\mathrm{OHB}$ causa una rápida y significativa vasoconstricción, compensada por el incremento en el oxígeno transportado y el flujo microvascular en los tejidos isquémicos ${ }^{6}$. Estos tejidos comprometidos se benefician del flujo del que son privados a los territorios sanos, fenómeno conocido como efecto Robin Hood. La vasoconstricción reduce el edema postraumático lo que contribuye al tratamiento del síndrome compartimental, las lesiones por aplastamiento y por quemaduras. La cicatrización de las heridas puede facilitarse por la amplificación del gradiente de oxígeno a lo largo de la periferia de los tejidos isquémicos y favorece la formación de la matriz de colágeno necesaria para la angiogénesis ${ }^{7}$. El aumento de la producción de especies reactivas de oxígeno (ROS) y de nitrógeno (RNS) pueden explicar otros efectos ${ }^{8}$ : facilita la acción del sistema de peroxidasas leucocitarias dependiente de oxígeno ${ }^{9}$, oxida las membranas lipídicas y las proteínas, daña el DNA e inhibe el metabolismo bacteriano. La OHB es especialmente eficaz frente a gérmenes anaerobios, dificulta la producción de exotoxinas y esporas clostridiales ${ }^{10}$, mata anaerobios como Clostridium perfringens, e inhibe el crecimiento de otras bacterias ${ }^{11}$.

En resumen la OHB tiene efectos hemodinámicos, acciones sobre la inmunidad y el transporte de oxígeno. El espectro de sus efectos terapéuticos va desde la reducción de la hipoxia y el edema hasta la mejora de la respuesta del huésped frente a la infección y la isquemia.

Con la excepción del neumotórax, no tratado, no existe una contraindicación absoluta para el tratamiento con OHB. Las contraindicaciones relativas incluyen la enfermedad pulmonar obstructiva crónica, la presencia de bullas ${ }^{12}$, las infecciones recientes de las vías respiratorias, oído o sinusitis, la cirugía torácica reciente y la claustrofobia. El embarazo es una contraindicación que no impide la OHB en los casos de intoxicación grave por $\mathrm{CO}^{13}$. El riesgo de toxicidad por oxígeno del sistema nervioso central (SNC) puede ser mayor en pacientes con antecedentes de epilepsia ${ }^{14}$, si bien la incidencia de complicaciones en estos pacientes o en los sometidos a cirugía reciente del SNC no se ha evaluado con exactitud. Existen estudios en animales sobre los efectos adversos en pacientes tratados concomitantemente con citostáticos como doxirrubicina o bleomicina, aunque los resultados no son concluyentes en estos pacientes, es aconsejable una especial valoración del riesgo/beneficio de la terapia con $\mathrm{OHB}^{15,16}$.

En general el tratamiento con OHB es bien tolerado ${ }^{17}$. La complicación más frecuente, observada en un $20 \%$ de los pacientes, es la miopía reversible por toxicidad directa sobre el cristalino. El barotrauma ótico sintomático aparece en un 2-4\% de los pacientes, sin embargo la ruptura de la membrana timpánica es excepcional. La toxicidad pulmonar crónica por oxígeno, o efecto Lorrain-Smith, es más frecuente en los pacientes sometidos a múltiples sesiones de tratamiento. Las convulsiones por toxicidad aguda del SNC son raras, pero pueden ser graves en las condiciones que se producen. Su incidencia se estima en 2,4 por cada 100.000 tratamientos $^{18} \mathrm{y}$ es más frecuente en pacientes tratados con glucocorticoides, hormona tiroidea, simpaticomiméticos e insulina, la hipoglucemia debe considerarse en el diagnóstico diferencial. La enfermedad descompresiva se produce por el nitrógeno contenido en el aire respirado a una presión superior a la atmosférica, por esto no es posible que se produzca si se respira oxígeno al 100\%. Durante las sesiones, como medida complementaria de seguridad, se realiza la descompresión gradualmente con el fin de evitar barotraumas. 


\section{Patologías tratadas con oxigenoterapia hiperbárica en el Hospital Central de la Defensa}

Tabla 1. Indicaciones aprobadas para OHB por la Health Care Financing Administration.
1. Embolismo gaseoso o aéreo
2. Intoxicación por monóxido de carbono
3. Accidentes de descompresión
4. Mionecrosis por Clostridium
5. Síndromes de aplastamiento y otras formas de isquemia traumática
6. Heridas y úlceras de difícil curación incluidas las producidas en el pie diabético
7. Anemia grave sin posibilidad de transfundir como en el caso de Testigos de Jehová
8. Abscesos cerebrales por Actinomyces
9. Infecciones necrotizantes de tejidos blandos
10. Osteomielitis crónica refractaria
11. Necrosis de hueso y tejidos blandos tras tratamiento con radioterapia
12. Injertos y colgajos de piel comprometidos

Los diferentes efectos producidos por las OHB facilitaron que entre sus recomendaciones se incluyeran un gran número de indicaciones, algunas sin suficiente validación científica con la consiguiente controversia respecto a su uso ${ }^{19}$. En las últimas décadas dos asociaciones, la Sociedad Americana de Submarinismo y Medicina Hiperbárica (UHMS) y el Comité Europeo para la Medicina Hiperbárica (ECHM), han tenido como objetivo establecer el uso médico de la OHB. Por ello se han utilizado sistemas para evaluar la evidencia científica disponible y sentar el nivel de la recomendación, como el del colegio americano de cardiología ${ }^{20} \mathrm{u}$ otros como el GRADE ${ }^{21}$. Este tipo de métodos son aplicables cuando existen estudios aleatorios controlados con un elevado grado de validez científica, en el caso de la OHB hay que reconocer que la realización de este tipo de ensayos es complicado por razones tales como la dificultad de efectuar trabajos sobre intervenciones terapéuticas aisladas como en las intoxicaciones por $\mathrm{CO}$ o accidentes de buceo, o sobre enfermedades que tienen una complejidad tal que hace muy difícil diseñar un estudio con un gran número de variables. Por estos motivos las indicaciones de la OHB son fruto del consenso entre expertos y sus conclusiones son comunicadas tras las reuniones periódicas de estas sociedades ${ }^{22-24}$. En la tabla 1 se reflejan las indicaciones aprobadas para la $\mathrm{OHB}$ en EEUU por la Health Care Financing Administration.

\section{OBJETIVOS}

Conocer las patologías de los pacientes tratados con $\mathrm{OHB}$ en la Comunidad Autónoma de Madrid (CAM) y los trabajos científicos que las avalan.

\section{MATERIAL Y MÉTODOS}

La población objeto de este estudio, observacional, descriptivo y transversal, la constituyen los pacientes tratados con $\mathrm{OHB}$ entre Febrero de 2013 y Julio de 2014 en el Servicio de MSB del HCD. Desde Febrero de 2013 esta cámara es la de referencia para los Hospitales de del Servicio Madrileño de Salud. Se revisaron las historias clínicas de los pacientes atendidos y se recogieron las siguientes variables. a) Número de pacientes enviados para tratamiento desde los Hospitales de la CAM.

b) Enfermos aceptados y rechazados para el tratamiento por el Servicio de MSB. Se registró la causa por la que se desestimó el tratamiento.

c) Edad y sexo de los pacientes tratados.

d) Hospitales de referencia de los pacientes tratados.

e) Patología por la que se indicaba desde el Hospital de Referencia del paciente el tratamiento en la cámara hiperbárica.

f) Complicaciones observadas en relación con la OHB.

\section{RESULTADOS}

Registramos 135 pacientes, tras excluirse 10 de los casos por falta de alguno de los datos exigidos. Se desestimaron para tratamiento 22 pacientes de los 135 (16,30\%): Dos de los casos presentaban una evolución favorable de la patología por la que se indicó la OHB por lo que se acordó no administrar este tratamiento. Uno de los pacientes presentaba una recidiva del proceso neoplásico causante de la complicación que había sentado su indicación. En 7 pacientes se desestimó el tratamiento por enfermedades concurrentes: 1 paciente esquizofrénico, 1 paciente con traqueotomía para el que no se disponía en ese momento de material apropiado para su tratamiento, 1 paciente tetrapléjico con incapacidad para realizar maniobras de compensación y no ser procedente en ese momento la miringocentesis, 2 pacientes con riesgo de sobre-expansión pulmonar por su patología respiratoria, 2 pacientes con patología del oído que no permitía su tratamiento en la cámara. Se excluyeron del tratamiento a 9 pacientes por considerarse que se trataban de indicaciones atípicas o bien porque las lesiones que presentaban hacían previsible que no fueran a mejorar con el tratamiento. Tres pacientes se rechazaron por estar en tratamiento con quimioterapia.

La muestra de este estudio es por tanto de 113 enfermos. El $60,18 \%$ hombres $(n=68)$ y el $39,82 \%$ mujeres $(n=45)$. Edad media 64,72 años, rango de 26 a 91 años.

Los pacientes tratados se recibieron de 15 Hospitales de la CAM. En la tabla 2 se muestra la distribución de pacientes por hospitales.

En el 52,21\% de los casos $(n=59)$ la indicación estuvo motivada por complicaciones del tratamiento con RT. La princi- 
Tabla 2. Hospitales de procedencia de los pacientes tratados.

\begin{tabular}{|lc|}
\hline Hospital de procedencia & N. $^{\mathbf{0}}$ casos \\
\hline HCD & 26 \\
Hospital Clínico San Carlos & 19 \\
Hospital de la Princesa & 14 \\
Hospital Severo Ochoa & 13 \\
Hospital Ramón y Cajal & 9 \\
Hospital Fundación Jiménez Díaz & 8 \\
Hospital Gregorio Marañón & 6 \\
Hospital Fuenlabrada & 5 \\
Hospital La Paz & 3 \\
Hospital Puerta Hierro & 3 \\
Hospital 12 de Octubre & 2 \\
Otros & 5 \\
\hline
\end{tabular}

Tabla 3. Patologías tratadas con $\mathrm{OHB}$ en el HCD.

\begin{tabular}{|lcc|}
\hline Indicación de la $\mathbf{O H B}$ & $\begin{array}{c}\mathbf{N}^{\mathbf{o}} \mathbf{d e} \\
\text { casos }\end{array}$ & Porcentaje \\
\hline Lesiones radio-inducidas & 59 & 52,2 \\
- ORNM & 18 & 15,9 \\
- Partes blandas & 14 & 12,4 \\
- Cistitis y proctitis & 26 & 23,0 \\
- SNC & 1 & 0,9 \\
Úlceras y heridas & 26 & 23,0 \\
Necrosis mandibular por & 8 & 7,08 \\
bifosfonatos & 6 & 5,31 \\
Osteomielitis crónica refractaria & 4 & 3,54 \\
Accidentes de buceo & 4 & 3,54 \\
Patología digestiva & 6 & 5,31 \\
Otras & 113 & 100 \\
\hline Total & & \\
\hline
\end{tabular}

pal de ella la ORNM en tumores de cabeza y cuello, 18 casos. Entre las lesiones de partes blandas por RT incluimos aquellas que afectaban a piel, mucosas y músculos, en nuestro caso 14 enfermos recibieron $\mathrm{OHB}$ por este motivo. La cistitis y proctitis radical es una complicación frecuente de la RT indicada en tumores de próstata, vejiga y útero, en 12 pacientes se indicó OHB por cistitis, en 11 por proctitis y en 3 por la asociación de estas dos complicaciones. En un enfermo se indicó OHB por afectación del SNC tras RT por un astrocitoma cerebral.

En el $23 \%$ de los casos $(n=26)$ se indicó OHB por úlceras y heridas de evolución tórpida con mala respuesta a tratamiento convencional. En el grupo las úlceras destacaban por su frecuencia las asociadas a pie diabético y las úlceras varicosas, 4 casos para cada una de estas causas, además se registraron otras etiologías: úlceras por isquemia, digitales asociadas a esclerodermia ( 2 casos) o un caso de úlcera de Martorel. Entre las heridas de evolución tórpida se contabilizaron 5 casos de cierre defectuoso de la herida quirúrgica tras injerto cutáneo.

Se indicó OHB en ocho enfermos que presentaban necrosis mandibular tras tratamiento con bifosfonatos. En seis enfermos se indicó por osteomielitis crónica refractaria al tratamiento convencional. Se registraron cuatro accidentes de buceo. Se indicó $\mathrm{OHB}$ en complicaciones digestivas de causa variada: 2 casos de fístula rectal por enfermedad de Crohn y 2 por úlcera rectal secundaria a colitis inespecífica. En el resto de los casos la indicación estuvo motivada por las siguientes patologías: dos casos de cistitis hemorrágica tras quimioterapia, un caso de gangrena de Fournier, un caso de isquemia aguda del glande, un caso de mucormicosis rinoorbitaria y un caso de sordera súbita.

En la tabla 3 se muestra la distribución de las indicaciones de la OHB de nuestro estudio.

En la mayoría de los casos se programaron 40 sesiones, todas a 2,5 ATA.

El tratamiento se suspendió en 8 pacientes $(7,14 \%)$ por mala tolerancia o complicaciones del mismo: 6 pacientes presentaron un barotrauma auditivo leve, 1 paciente por una edematización del miembro en el que presentaba la lesión, en otro se suspendió por claustrofobia.

\section{DISCUSIÓN}

Las publicaciones sobre la OHB se han multiplicado en los últimos años con especial atención a sus indicaciones ${ }^{25}$, quizás el campo de mayor controversia de este tratamiento. Por este motivo, creemos de interés conocer las patologías tratadas en el Servicio de MSB del HCD y revisar la evidencia científica en estas indicaciones.

La cámara hiperbárica del HCD es la de referencia para administrar OHB en la CAM desde Febrero de 2013. No hemos encontrado artículos en los que se exponga la actividad desarrollada en la cámara hiperbárica de un centro hospitalario, tras la búsqueda en PubMed con los términos "hyperbaric", "oxygen", "therapy".

En general el tratamiento es bien tolerado, prácticamente exento de complicaciones, y las que obligan a suspenderlo suelen ser de carácter leve, entre estas destacan los problemas de compensación.

Más de la mitad de nuestros enfermos recibieron OHB como tratamiento de los efectos secundarios de la RT. Se estima que a un $50 \%$ de los pacientes diagnosticados de cáncer se les administrará RT en algún momento de su enfermedad y que un $5 \%$ de ellos pueden presentar complicaciones derivadas de sus efectos, algunas tras un periodo de latencia que puede oscilar desde meses a varios años. Sus secuelas se relacionan con la hipoxia tisular secundaria al daño del endotelio que causa inflamación, fibrosis y finalmente necrosis tisular que se traducen en la aparición de endarteritis obliterante. Aunque estas consecuencias raramente comprometen la vida, sí producen un deterioro de la calidad de vida de los pacientes.

La ORNM, en los pacientes con tumores de cabeza y cue1lo, ha sido la causa más frecuente dentro de este grupo. La mandíbula es un hueso pobremente vascularizado y de gran densidad por lo que es muy sensible a los efectos de la radiación. Existen 14 ensayos que informan de los beneficios de la $\mathrm{OHB}$ en el tratamiento de la osteoradionecrosis mandibular, si bien sólo uno de ellos es un estudio controlado y aleatorizado de una muestra pequeña en el que no se detallan la asignación al azar o como se valoran los de resultados ${ }^{26}$. Un solo estudio no presenta resultados favorables ${ }^{27}$, en estos casos la $\mathrm{OHB}$ se 


\section{Patologías tratadas con oxigenoterapia hiperbárica en el Hospital Central de la Defensa}

administraba después de realizar el tratamiento quirúrgico de la necrosis, mientras que en algunos los ensayos con resultados positivos se insiste en la importancia de recibir OHB previa al tratamiento quirúrgico de la necrosis mandibular ${ }^{28}$. La ORNM es una indicación aceptada por los expertos en OHB como indiscutible.

La indicación de OHB por lesiones de partes blandas secundarias a RT es la segunda en frecuencia en el grupo de pacientes tratados con RT. La OHB se ha dirigido sobre tres tipos de lesiones diferentes: 1) Las producidas tras la radiación de tumores de cabeza y cuello, sobre todo la necrosis laríngea. Un estudio prospectivo no aleatorizado muestra los beneficios de la $\mathrm{OHB}$ en la reducción de la dehiscencia de sutura y del riesgo de infección tras RT en los tumores de cabeza y cuello ${ }^{29}$ tratados quirúrgicamente, otros 5 estudios de series pequeñas de casos informan de los resultados beneficiosos de la $\mathrm{OHB}^{25}$. 2) En las de la pared torácica y mama se ha publicado una serie de casos que apoya el tratamiento complementario con $\mathrm{OHB}$ en la necrosis de tejidos blandos inducidas por la radiación, aunque la OHB deben de acompañarse de un desbridamiento adecuado que incluya la extirpación del hueso necrótico para asegurar la resolución de la necrosis ósea además de la de los tejidos blandos ${ }^{30}$. Un estudio no aleatorizado, comparativo entre un grupo de enfermas que recibieron OHB por secuelas de RT por cáncer de mama frente a otro que no lo recibió mostraba una reducción de los signos inflamatorios ${ }^{31}$. 3) En las de pelvis y pared abdominal los trabajos publicados incluyen series de casos que informan del probable beneficio de la OHB en las lesiones secundarias a RT en esta localización ${ }^{32}$.

La cistitis rádica fue la tercera indicación más frecuente entre los pacientes con lesiones radio inducidas. A pesar de que son muchas las publicaciones sobre el uso de OHB en esta complicación, ninguno de los estudios es randomizado o controlado. La mayoría son revisiones retrospectivas o serie de casos en los que la $\mathrm{OHB}$ es usada como un tratamiento se segunda línea tras el fracaso o mejoría incompleta con el habitual. Existen dos estudios prospectivos, que apoyan el uso de la $\mathrm{OHB}^{33,34}$, en el más reciente de ellos se utilizó la OHB como tratamiento de primera línea con buen resultado. Hoy en día es una de las indicaciones aceptadas sin reservas en los centros que disponen de cámara hiperbárica y en la experiencia de los enfermos tratados en nuestro Hospital una de patologías que mejor responden al tratamiento.

La proctitis rádica supuso una indicación habitual de $\mathrm{OHB}$ en la muestra que presentamos. Las publicaciones que avalan su uso son series de casos que comunican su efecto beneficio$\mathrm{SO}^{25}$. Los resultados en los pacientes tratados en la muestra son muy favorables aunque en nuestra experiencia se precisan un número mayor de sesiones que en los afectados por cistitis rádica.

Uno de los casos tratados corresponde a las lesiones radioinducidas en el SNC tras RT por un astrocitoma. Son pocos los estudios realizados sobre esta indicación, la serie más numerosa es la de 10 pacientes pediátricos en los que se comunicaba la mejoría inicial de las lesiones y dicha mejoría se mantenía en el tiempo en 5 de ellos ${ }^{35}$.

Los enfermos con úlceras y heridas de evolución tórpida con mala respuesta tras tratamiento convencional son el segun- do grupo en frecuencia de nuestra muestra. Es un conjunto de pacientes con lesiones de diferente etiología en el que destacan algunos tipos. Las úlceras en el pie diabético y las varicosas fueron las más habituales. Una revisión sistemática ${ }^{36}$ sobre el valor de la $\mathrm{OHB}$ en las lesiones del pie diabético y la prevención de la amputación, incluye siete ensayos clínicos randomizados de los que dos destacan por su calidad metodológica, esta revisión concluye que existen evidencias sobre la utilidad del tratamiento de la OHB en lesiones con isquemia asociada, si bien se reconoce que deberían realizarse estudios con muestras más amplias y con validez suficiente para justificarla como un tratamiento habitual. En el caso de las úlceras por insuficiencia venosa la evidencia se reduce a un ensayo randomizado, con una muestra pequeña, que apoya el uso de OHB como un tratamiento complementario al convenciona ${ }^{37}$. El empleo de OHB en otros tipos de úlceras se apoya en publicaciones de casos aislados o series de $\operatorname{casos}^{38,39}$. Entre las heridas de evolución tórpida destacan en nuestra muestra 5 pacientes tratados con un injerto cutáneo, un estudio con una serie de casos informa de los beneficios de la OHB si bien no se disponen de ensayos aleatorizados doble ciego ${ }^{40}$.

La osteonecrosis mandibular por bifosfonatos es una patología emergente y de difícil tratamiento. La OHB se ha utilizado frente a esta complicación en un estudio piloto ${ }^{41}$ en el que se informan los beneficios de la misma cuando se asocia de forma adyuvante y siempre que se suspenda el tratamiento con bifosfonatos. En nuestra muestra supone el tercer grupo más frecuente de pacientes.

La osteomielitis crónica refractaria ha sido tratada con OHB en muchos hospitales desde hace años, no se dispone de estudios randomizados, aunque las publicaciones de series de $\operatorname{casos}^{42,43}$ apoyan su uso cuando se asocia a un tratamiento médico y quirúrgico correcto. En nuestra muestra 5 pacientes fueron tratados por esta patología.

Sólo se registraron 4 tratamientos por secuelas tras accidentes de buceo, un dato que se explica por la localización de la cámara en una zona alejada del litoral que hace poco probable la presentación de casos agudos. Aunque se carece de estudios randomizados, la $\mathrm{OHB}$ es reconocida como el tratamiento de elección en los protocolos de la enfermedad descompresiva ${ }^{44}$. El efecto explicado por la ley de Boyle se considera una base firme para sentar dicha indicación.

Los estudios sobre OHB en la enfermedad inflamatoria incluyen publicaciones sobre series de casos de enfermedad de Crohn y colitis ulcerosa. Una revisión sistemática ${ }^{45}$ de 2014 concluye que la OHB es una opción de tratamiento relativamente seguro y teóricamente eficaz en estos pacientes, aunque hasta el momento se carece de ensayos aleatorizados.

En el resto de los casos tratados, sus indicaciones se apoyan en estudios de diferente entidad: la retinitis pigmentosa en estudios randomizados de casos-control ${ }^{46}$, la sordera súbita neurosensorial en series de casos en los que se compara el uso de $\mathrm{OHB}$ con otros tratamientos ${ }^{47}$, la cistitis hemorrágica por ciclofosfamida en estudios de casos aislados ${ }^{48}$ y en la enfermedad de Fournier y la mucormicosis como una recomendación aceptada tras lo comunicado en en el tratamiento de casos ais$\operatorname{lados}^{49,50}$ que puede considerarse tras lo aportado por algunas publicaciones $^{51}$. 


\section{CONCLUSIONES}

1. Las indicaciones de $\mathrm{OHB}$ en nuestra muestra comprende un amplio número de patologías.

2. Las lesiones radio inducidas son la indicación más frecuente de los enfermos tratados con OHB en el HCD. En este grupo destaca la ORNM tras RT en tumores de cabeza y cuello.

3. Todas las indicaciones de OHB de la muestra presentada cuentan con estudios que avalan su uso aunque no existen, para ninguna de ellas, estudios randomizados controlados doble ciego.

4. Las complicaciones observadas con el tratamiento con $\mathrm{OHB}$ en nuestra muestra son leves por lo que la podemos considerar un tratamiento seguro.

5. Creemos que la OHB ofrece una gran oportunidad para investigar la evidencia científica firme que avale sus indicaciones.

\section{BIBLIOGRAFÍA}

1. Kot J, Desola J, Simao AG, Gough-Allen R, Houman R, Meliet JL, Galland F, Mortensen C, Mueller PH, Sippinen S. A European code of good practice for hyperbaric oxygen therapy.Int Marit Health. 2004;55(1-4): 121-30.

2. Desola J. Bases y fundamento terapéutico de la oxigenoterapia hiperbárica Revista virtual de medicina hiperbárica. En http://www.cccmh.com/REVISTA-OHB/Revista-OHB.htm

3. Leach RM, Rees PJ, Wilmshurst P. Hyperbaric oxygen therapy. BMJ 1998; 317 (7166):1140-3

4. Ernst A, Zibrak JD. Carbon monoxide poisoning. $N$ Engl J Med. 1998;339(22):1603.

5. Gill AL, Bell CN.Hyperbaricoxygen: its uses, mechanisms of action and outcomes.QJM. 2004 Jul;97(7):385-95.

6. Nylander G, Lewis D, Nordström H, Larsson J. Reduction of postischemic edema with hyperbaric oxygen. Plast Reconstr Surg. 1985;76(4):596.

7. Roth RN, Weiss LD Hyperbaric oxygen and wound healing. Clin Dermatol. 1994; 12(1):141.

8. Thom SR. Hyperbaric oxygen: its mechanisms and efficacy. Plast Reconstr Surg. 2011; 127 Suppl 1:131-41.

9. Knighton DR, Halliday B, Hunt TK. Oxygen as an antibiotic.A comparison of the effects of inspired oxygen concentration and antibiotic administration on in vivo bacterial clearance. Arch Surg. 1986 Feb;121(2):191-195.

10. Kaye D. Effect of hyperbaric oxygen on Clostridia in vitro and in vivo. Proc Soc Exp Biol Med. 1967;124(2):36

11. Mader JT, Brown GL, Guckian JC, Wells CH, Reinarz JA. A mechanism for the amelioration by hyperbaric oxygen of experimental staphylococcal osteomyelitis in rabbits. J Infect Dis. 1980;142(6):915.

12. Toklu AS, Korpinar S, Erelel M, Uzun G, Yildiz S. Are pulmonary bleb and bullae a contraindication for hyperbaric oxygen treatment?-. Respir Med. 2008 Aug;102(8):1145-47.

13. Ernst A, Zibrak JD. Carbon monoxide poisoning. $\mathrm{N}$ Engl J Med. 1998;339(22):1603.

14. Roth RN, Weiss LD. Hyperbaric oxygen and wound healing.ClinDermatol. 1994;12(1):141.

15. Torp KD, Carraway MS, Ott MC, Stolp BW, Moon RE, Piantadosi CA, Freiberger Safe administration of hyperbaric oxygen after bleomycin: a case series of 15 patients. JJ. Undersea Hyperb Med. 2012 Sep;39(5):873-79.

16. Karagoz B, Suleymanoglu S, Uzun G, et al. Hyperbaric oxygen therapy does not potentiate doxorubicin-induced cardiotoxicity in rats. Basic ClinPharmacolToxicol 2008; 102:287.

17. Plafki C, Peters P, Almeling M, Welslau W, Busch R. Complications and side effects of hyperbaric oxygen therapy. Aviat Space Environ Med. 2000;71(2):119.
18. Yildiz S, Aktas S, Cimsit M, et al. Seizure incidence in 80,000 patient treatments with hyperbaric oxygen. Aviat Space Environ Med 2004; 75:992.

19. Gabb G, Robin ED. Hyperbaric oxygen: a therapy in search of disease. Chest 1987; 92:1074-82.

20. Tricoci P, Allen JM, Kramer JM, Califf RM, Smith SC Jr. Scientific evidence underlying the ACC/AHA clinical practice guidelines.JAMA.2009;301:831-41.

21. M.H. Murad et al. Using GRADE for evaluating the quality of evidence in hyperbaric oxygen therapy clarifies evidence limitations. Journal of Clinical Epidemiology 2014; 67: 65-72.

22. 7th European Consensus Conference on Hyperbaric Medicine, Lille, France, December, 2004. Recommendations of the Jury. In: Marroni A, Mathieu D, Wattel F, editors. The ECHM Collection. Volume 3. Flagstaff, AZ: Best Publishing Company; 2008. p. xv-xxix.

23. Jacek Kot and Daniel Mathieu.Controversial issues in hyperbaric oxygen therapy: a EuropeanCommittee for Hyperbaric Medicine Workshop report Diving and Hyperbaric Medicine Volume 41 No. 2 June 2011.

24. https://www.modahealth.com/pdfs/med_criteria/HyperbaricOxygen.pdf.

25. Lee $\mathrm{CH}$, Lee L, Yang KJ, Lin TF.Top-cited articles on hyperbaric oxygen therapy published from 2000 to 2010. Undersea Hyperb Med. 2012 NovDec;39(6):1089-98.

26. Feldmeier JJ. Hyperbaricoxygen therapy and delayed radiation injuries (soft tissue and bony necrosis). Undersea Hyperb Med. 2012 NovDec;39(6):1121-39.

27. Maier A, Gaggl A, Klemen H, Santler G, Anegg U, Fell B, Kärcher H, Smolle-Jüttner FM, Friehs GB. Review of severe osteoradionecrosis treated by surgery alone or surgery with postoperative hyperbaric oxygenation. Br J Oral Maxillofac Surg. 2000 Jun;38(3):173-6.

28. Cronje FJ. A review of the Marx protocols: prevention and management of osteoradionecrosis by combining surgery and hyperbaric oxygen therapy. SADJ. 1998 Oct; 53(10):469-71.

29. Marx RE.Radiation injury to tissue. In: Kindwall EP, ed. Hyperbaric Medicine Practice, Second Edition. Flagstaff, Best Publishing, 1999, pp 665-740.

30. Feldmeier JJ, Heimbach RD, Davolt DA, Court WS, Stegmann BJ, Sheffield PJ. Hyperbaric oxygen as an adjunctive treatment for delayed radiation injury of the chest wall: a retrospective review of twenty-three cases. Undersea Hyperb Med. 1995 Dec;22(4):383-93.

31. Carl UM, Feldmeier JJ, Schmitt G, Hartmann KA.Hyperbaric oxygen therapy for late sequelae in women receiving radiation after breast conserving surgery.Int J RadiatOncolBiolPhys 2001;49:1029-31.

32. Feldmeier JJ, Heimbach RD, Davolt DA, Court WS, Stegmann BJ, Sheffield PJ. Hyperbaric oxygen as an adjunctive treatment for delayed radiation injuries of the abdomen and pelvis. Undersea Hyper Med 1997;23(4):205-13.

33. Bevers RF, Bakker DJ, Kurth KH. Hyperbaric oxygen treatment for haemorrhagic radiation cystitis.Lancet 1995;346: 803-5.

34. Oscarsson N, Arnell P, Lodding P, Ricksten SE, Seeman-LoddingH.Hyperbaric oxygen treatment in radiation-induced cystitis and proctitis: a prospective cohort study on patient-perceived quality of recovery.Int J RadiatOncolBiol Phys. 2013 Nov 15;87(4):670-5.

35. Chuba PJ, Aronin P, Bhambhani K, Eichenhorn M, Zamarano L, Cianci P, Muhlbauer M, Porter AT, Fontanesi J. Hyperbaric oxygen therapy for radiation-induced brain injury in children. Cancer 1997;80:2005-2012.

36. Stoekenbroek RM, Santema TB, Legemate DA, Ubbink DT, van den Brink A, Koelemay MJ. Hyperbaricoxygen for the treatment of diabetic foot ulcers: a systematic review. Eur J VascEndovasc Surg. 2014 Jun;47(6):647-55.

37. Hammarlund C, Sundberg T. Hyperbaric oxygen reduced size of chronic leg ulcers: a randomized double-blind study. Plast Reconstr Surg. 1994 Apr; 93(4):829-33.

38. Markus YM, Bell MJ, Evans AW. Ischemic scleroderma wounds successfully treated with hyperbaric oxygen therapy. J Rheumatol. 2006 Aug;33(8):1694-6.

39. Frada G, Di Chiara A, Nicolosi G, Alletto M, Barbagallo Sangiorgi G. A case of Martorell's ulcer: considerations on the use of hyperbaric oxygenotherapy.Angiologia. 1989 May-Jun;41(3):93-5.

40. Larson JV, Steensma EA, Flikkema RM, Norman EM. The application of hyperbaric oxygen therapy in the management of compromised flaps. Undersea Hyperb Med. 2013 Nov-Dec;40(6):499-504.

41. Freiberger JJ, Padilla-Burgos R, Chhoeu AH, Kraft KH, Boneta O, Moon RE, Piantadosi CA. Hyperbaric oxygen treatment and bisphosphonate-induced osteonecrosis of the jaw: a case series. J Oral Maxillofac Surg. 2007 Jul;65(7):1321-7. 


\section{Patologías tratadas con oxigenoterapia hiperbárica en el Hospital Central de la Defensa}

42. Chen CE, Ko JY, Fu TH, Wang CJ. Results of chronic osteomyelitis of the femur treated with hyperbaric oxygen: a preliminary report. Chang Gung Med J. 2004 Feb;27(2):91-7.

43. Chen CE, Shih ST, Fu TH, Wang JW, Wang CJ. Hyperbaric oxygen therapy in the treatment of chronic refractory osteomyelitis: a preliminary report. Chang Gung Med J. 2003 Feb;26(2):114-21.

44. Vann RD, Butler FK, Mitchell SJ, Moon RE. Decompression illness. Lancet. 2011 Jan 8;377(9760):153-64.

45. Dulai PS, Gleeson MW, Taylor D, Holubar SD, Buckey JC, Siegel CA. Systematic review: The safety and efficacy of hyperbaric oxygen therapy for inflammatory bowel disease. Aliment Pharmacol Ther. 2014 Jun;39(11):126675.

46. Vingolo EM, Rocco M, Grenga P, Salvatore S, Pelaia P. Slowing the degenerative process, long lasting effect of hyperbaric oxygen therapy in retinitis pigmentosa. Arch Clin Exp Ophthalmol. 2008 Jan;246(1):93-8.
47. Yang $\mathrm{CH}, \mathrm{Wu} \mathrm{RW}$, Hwang CF Comparison of intratympanic steroid injection, hyperbaric oxygen and combination therapy in refractory sudden sensorineural hearing loss. Otol Neurotol. 2013 Oct;34(8):1411-6.

48. Davis M, MacDonald H, Sames C, Nand K. Severe cyclophosphamide-induced haemorrhagic cystitis treated with hyperbaric oxygen. N Z Med J 2011; 124: 48-54.

49. Mallikarjuna MN, Vijayakumar A, Patil VS, Shivswamy BS. Fournier's Gangrene: Current Practices. ISRN Surg. 2012;2012:942437. doi: 10.5402/2012/942437. Epub 2012 Dec 3.

50. Lin SY, Lu PL, Tsai KB, Lin CY, Lin WR, Chen TC, Chang YT, Huang CH, Chen CY, Lai CC, Chen YH. A mucormycosis case in a cirrhotic patient successfully treated with posaconazole and review of published literature. Mycopathologia. 2012 Dec;174(5-6):499-504.

51. Riseman JA., Zamboni WA, Curtis A., Graham DR., Konrad HR., Ross DS Hyperbaric oxygen therapyfor necrotizing fasciitis reduces mortality and the need fordebridements. Surgery 1990; 108(5): 847-850. 